Marie-Eve Thérenty et Adeline Wrona (dir.). 2020. L'écrivain comme marque (Paris : Sorbonne Université Presses)

\title{
Jürgen Siess
}

\section{(2) OpenEdition}

Journals

Electronic version

URL: http://journals.openedition.org/aad/5377

DOI: $10.4000 /$ aad. 5377

ISSN: 1565-8961

\section{Publisher}

Université de Tel-Aviv

Electronic reference

Jürgen Siess, "Marie-Eve Thérenty et Adeline Wrona (dir.). 2020. L'écrivain comme marque (Paris :

Sorbonne Université Presses)", Argumentation et Analyse du Discours [Online], 26 | 2021, Online since 14 April 2021, connection on 16 April 2021. URL: http://journals.openedition.org/aad/5377 ; DOI: https:// doi.org/10.4000/aad.5377

This text was automatically generated on 16 April 2021.

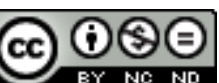

Argumentation \& analyse du discours est mis à disposition selon les termes de la licence Creative Commons Attribution - Pas d'Utilisation Commerciale - Pas de Modification 4.0 International. 


\title{
Marie-Eve Thérenty et Adeline Wrona (dir.). 2020. L'écrivain comme marque (Paris : Sorbonne Université Presses)
}

\author{
Jürgen Siess
}

\section{REFERENCES}

Marie-Eve Thérenty et Adeline Wrona (dir.). 2020. L'écrivain comme marque (Paris :

Sorbonne Université Presses), 244 p., ISBN 9791023106480

1 L'écrivain comme marque, ouvrage collectif dirigé par Marie-Eve Thérenty et Adeline Wrona, traite du «travail du branding autour du nom d'écrivain » (7). On y étudie des acteurs de l'édition et des médias les plus divers, allant de la dynastie des Dumas au dixneuvième siècle jusqu'aux auteurs 'profitant' de la publicité qu'offre Internet. Sont ainsi présentés des pans très divers du développement du marché littéraire. Comme forme la plus poussée du branding apparaît la transformation du nom d'auteur en nom d'une gamme de produits. - Plusieurs des contributions intéressent l'Analyse du Discours (AD), notamment celles qui reprennent le concept d'image de soi ou ethos.

Une première conclusion qui ressort des contributions est qu'on ne peut échapper aux lois du marché. Plusieurs des contributeurs semblent cependant vouloir échapper à une telle conclusion, lorsqu'ils/elles tâchent de favoriser la "valeur symbolique " au détriment de la «valeur commerciale». On trouve, par exemple, une occultation de la valeur marchande chez Sarah Mombert traitant des Dumas ou chez Yoan Vérilhac traitant du Symbolisme. Ceci n'empêche pas d'ailleurs les contributeurs en question d'être fascinés par les possibilités qu'offrent aux écrivains les médias les plus modernes. 
On touche là au cœur du problème. Des écrivains qui se livrent de leur gré aux éditeurs et publicitaires jusqu'à ceux/celles qui voudraient sortir de la " prison dorée » (Jérôme Meizoz, 164), tous se sentent tiraillés entre esthétique et économie, en clair: entre vouloir accéder au champ culturel - ou y garder leur place (champ où tout n'est que symbole), et être entraînés dans la sphère de circulation des marchandises (où tout est production de plus-value). Le cas de Tatiana de Rosnay est instructif ici : quoiqu'elle entende se situer dans l'avant-garde en utilisant les possibilités d'Internet, l'amateur de selfies gomme sa dépendance par rapport au système capitaliste - qu'en réalité elle salue et soutient (Valérie Jeanne-Perrier).

Le terme-clé des travaux présentés ici est la marque. Or, qui parle de marque et de marketing, ne devrait pas ignorer que tout dépend du marché, que «marché » est une notion fondamentale pour toute analyse où l'on entend prendre en considération le « contexte socio-économique» (Introduction, 7). Le contexte est, dans ce volume, le marché de l'édition (incluant les médias). Galia Yanoshevsky se distingue ici par rapport aux autres contributeurs en abordant directement la question : traitant du branding à l'exemple d'Amos $\mathrm{Oz}$, elle met sa réflexion clairement sous le signe du marketing et du processus de "facilitation et canalisation des échanges » (188).

On sait que le marché de l'édition fait partie du Marché, de la sphère de circulation des marchandises, même s'il a, dans cette sphère, une place distincte par rapport au marché du bétail ou du textile. Marx déjà avait noté à ce propos que l'artiste utilise ses propres moyens de production et « vend le produit de son travail comme marchandise qui lui appartient »- et que sa position entre l'ouvrier et le capitaliste l'amène à se piquer d'être impartial, plus proche du capitaliste que de l'ouvrier (Marx à Annenkow, 1846). - Comme certains des contributeurs l'ont (entre)vu - Matthieu Letourneux, Caroline Marty, Olivier Aïm et Anneliese Depoux, Adeline Wrona -, la reproduction massive et diversifiée des œuvres rendue possible par les technologies modernes a changé la donne : au dix-neuvième siècle l'écrivain devient autonome, au vingtième il en paye le prix, la production artistique devenant de plus en plus et ouvertement soumise aux lois du marché, aux conditions de la distribution capitaliste.

6 Le paradoxe de l'écrivain devenu autonome et valorisé par cette autonomisation, et de sa transformation en "écrivain-marque ", est pointé dans l'Introduction (7). Je suggérerais d'élargir, de prolonger ce paradoxe comme suit : l'éditeur-entrepreneur et les agents intermédiaires, en promettant à l'auteur de le gratifier d'un gain, lui tendent un piège - en fait, c'est l'entreprise qui la plupart du temps fait le plus grand profit aux dépens de l'écrivain-employé ; qui plus est, dans bien des cas, l'écrivain se tend luimême le piège. A l'appui, je citerais l'exemple de l'auteur qui produit un récit pour promouvoir les capsules Nespresso (Ambre Abid-Dalençon, 124) ou celui de l'écrivain qui, se prêtant au marketing, accepte le figement de son nom et de sa personne et qui abandonne l'autorité à la marque (C. Marty, 78 et 83). Je préciserais ici : l'autorité passe à l'éditeur-entrepreneur; en d'autres termes : la marque aliène le producteur de son produit.

7 Notons, à propos du piège où tombe plus d'un, une exception fabuleuse : Salvador Dali, comme le montre Ruth Amossy (172), joue sur toutes les possibilités qu'offre le marché capitaliste et réussit à devenir un redoutable concurrent des entrepreneurs. Mais, à mon avis, les quelques exceptions traitées dans ce volume (tels que Musso ou J.K. Rowling) ne font que confirmer la règle : le marché est plus puissant que le plus grand producteur de best-sellers ou de signes censés lui garantir la gloire. C'est le cas 
lorsqu'on montre, comme Sylvie Ducas (111), que «la valeur marchande occulte la valeur symbolique »: le poster géant (photo de l'auteur à taille réelle) ne fait que donner l'illusion que c'est l'écrivain qui tire le plus grand profit - je soulignerais que la plus-value revient d'abord à l'entreprise qui, si elle n'affiche que son nom, occupe en tout cas le premier rang.

Venons-en aux articles qui s'inspirent dans une plus ou moins grande mesure de l'AD. Meizoz, partant des notions d'ethos et de posture, remarque que de nos jours bien des écrivains se mettent en scène, et que, s'ils engagent leur corps et leur voix, cela sert davantage la promotion commerciale que leur reconnaissance comme producteurs littéraires. Houellebecq représente, à ses yeux, celui qui, lorsque son nom d'auteur devient "pur désignateur commercial» (164), ne voit d'autre solution que le repli romantique. A l'opposé on pourrait situer François Bon, que Florence Vinas-Thérond présente ici comme rétif au système: Bon se sert des possibilités d'Internet pour déstabiliser la marque (225) qui fige le travail de l'écrivain à des fins d'exploitation. Contre l'écrivain-marque il favorise l'artiste-amateur, qu'il considère comme expérimentateur et initiateur d'un ensemble d'écrivains ouvert aux lecteurs : il s'agit de construire "un ethos collectif de pionniers partageant [...] une éthique de la littérature à l'opposé du marketing » (234).

9 R. Amossy, quant à elle, s'intéresse à la tension entre valeur symbolique et valeur économique, et entre l'instance auctoriale et l'instance éditoriale. L'«image promotionnelle » se situerait entre l'image que projette l'auteur et celle que projette de lui l'éditeur; elle joue sur l'identification du consommateur avec le producteur par le biais de la marque (brand) «dotée d'une personnalité »: au nom de l'auteur doit s'attacher, dans la logique du marché, une "personnalité particulière » (169). La marque, pourrait-on préciser, est censée créer l'illusion que l'écrivain et son produit ne font qu'un, en d'autres termes: l'image de l'auteur est vouée à être absorbée par l'image de la marchandise (on pourrait rapprocher cette 'absorption' du phénomène de l'aliénation). - Amossy illustre sa thèse en évoquant la démarche très différente de deux écrivains. Dali entre dans le jeu, avec l'illusion qu'il sera le grand gagnant, tandis que, à l'autre extrême, Julien Gracq refuse toutes les possibilités offertes par le marché. Si Gracq se montre perspicace, cela n'empêche que «l'image épurée » qu'il construit à l'intention du lecteur ne peut se passer de «l'appareil éditorial» (174) et que, malgré lui, il dépend du marché. On rapprochera de cette analyse celle que M.-E. Thérenty consacre aux Editions Minuit et à ses écrivains : ici la «marque-éditeur » est opposée à la «marque-auteur ». En étudiant le rapport entre Jérôme Lindon et Samuel Beckett, elle montre que si la marque de l'auteur phare est effacée, cet effacement sert la marque visible de l'éditeur. Je préciserais que, là encore, c'est l'entrepreneur qui sort gagnant, sur qui rejaillit l'aura de celui qui en sa personne crée et cristallise une avantgarde (voir mon "Samuel Beckett's Posture in the French Literary Field", Beckett at 100, ed. by L. Ben-Zvi \& A. Moorjani, 2008).

10 Un cas particulier est analysé par Oriane Deseilligny: celui d'un ethos construit sur Facebook, où l'image est élaborée dans un jeu de miroir entre photo et fragments de texte, entre " conduites non-verbales » et construction discursive. Des auteures comme Fabienne Swiatly ou Fred Griot utiliseraient un dispositif façonné par une logique marchande comme Facebook pour imposer des gestes susceptibles de dénoncer le caractère rigide du dispositif, ou de défendre la cause d'une minorité. En élaborant à 
l'intention des internautes une image du métier d'écrivain caractérisé par le work in progress, l'auteure entend circonvenir le figement en auteur-marque.

11 On retiendra de cet ensemble de travaux de recherche l'intérêt du sujet qui inclut les développements les plus récents de l'édition et des médias, une introduction substantielle et plusieurs contributions notables (entre autres, Matthieu Letourneux faisant ressortir la logique capitaliste ou Florence Vinas-Thérond évoquant la possibilité de résister à la marchandisation). Si l'examen d'un riche ensemble de corpus et la recherche interdisciplinaire réalisée ici méritent toute notre attention, on peut cependant regretter que la démonstration ne s'appuie pas sur une hypothèse centrale (d'ordre sociologique, comme le laisse entendre l'Introduction), et qu'on ne voie pas toujours une ligne directrice susceptible de fédérer les différentes hypothèses avancées par les contributeurs. L'apport de ce travail aux facettes multiples aurait été d'autant plus important.

\section{AUTHORS}

\section{JÜRGEN SIESS}

Université de Caen, ADARR 